\title{
A Method for Accelerating CFG-I'arsing by Using Dependency Information
}

\author{
Hidco Watamabo \\ IBM Research, 'Iokyo Rosearch Isaboratory \\ 1623-14. Shimotsuruma, Yamato, Kanagawa 242-8502, Japan \\ walianabo(rotrl.ibm.co.jp
}

\begin{abstract}
Alsstract
'I'his paper describes an algorithm for accelerating the Cl(c-parsing process by using dependency (or: modifier-modifiec rolationship) information given by, for instance, dependency estimation prograns such as stoc]lastic parsers, usere's indication in an interactive application, and linguistic annotations adred in a source text. 'This is a method for en-

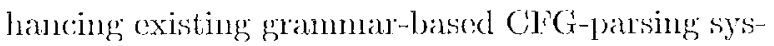
tom by using dependency information.
\end{abstract}

\section{Introduction}

The parsing systen is a kry component for natual language applications such as machine tanslation, information retrieval, text smmmarization, and its performance (processing; spoed and acenracy) is very inuportant, to the successs of these aptplications.

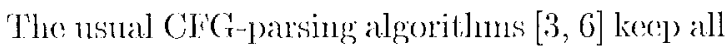
intermediate possibilities which may or may not be used in the final parse results. Therefore, we usually reduce these intermediate possibilities which are nulikely to be used as final results in the middle of the process by using several pruning technicpues. One good information source for pruning is dependency information between words. It hats not been so adsy to got such dependency information until a few yoars ago, but, the situation has recently changed.

Recent intensive studies on statistical approach $[7,1,2]$ advanced statistical parsing systems, and we can get relatively corroct, dependency information using these systems. Further, if we suppose an interactive NIP system, then there are some types of user interactions which can be considered to determine the modifiec candidate. In addition, recent studies on the linguistic information annotation $[10,4,12,13]$ provide tools by which a user can easily annotate linguistic information (special XMI, markup tags) into sonce texts, and wo can expect to see an increase of the number of texts with linguistic information. This linguistic information usually includes dependency information. lor instance, the following example shows an annotation example by Iinguistic Annotation Language described in $[12,13]$, and the id and mod attributes inside lal:w elements specify word dependencies.

Ho 〈lal:w id=" $1 "\rangle$ saw $/ /$ lal:w a man $\langle$ lal:w

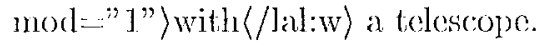

In this example, the word "with" modifies the word "saw."

As shown in the above examples, we can now ged dependency information more easily than a fow years ago. 'Ihis papor describes an algorithm for' accolerating CF( $\mathrm{x}$-parsing systems by using such dependency (or modifies-modifiee relationship) information. The proposed algorithm does not atssume all words alre given dependency information, rather it works in case such that some of words aro partially given dependency information.

\section{Optimizing Algorithm Using De- pendency Information}

We nse a normal CFG parsing system with one extension that for each sule there must be one right; hand side (or lRISS) tom ${ }^{1}$ marked as a head, and the information of a head term is transfored to the left-hand side (or LIIS) tom. In this paper, a ClC rule is demoted as follows:

$$
\left\{X \rightarrow Y_{1} \ldots Y_{i} * \ldots Y_{n}\right\}(n>0)
$$

In the above notation, $X$ is the left-hand side (or LISS) term, and $Y_{i}$ are right-hand side (or RISS) terms, and a RHS term followed by an astcrisk ${ }^{\prime *}$ ' is a head term. The typical usage of the head is that the IHAS term shares many features of the licad term in the RHS. For instance, a matiching word of the the LIIS term becomes the same as the one of the head term in the RHS.

For each rule, an are is constructed over a word segment in an input sentence. An arc is denoted using terms of its base rule as follows:

$$
\left[X \rightarrow Y_{1} \ldots Y_{i} \cdot Y_{i+1} * \ldots Y_{n}\right]
$$

\footnotetext{
${ }^{1}$ A term expresses a non-terminal symbol in Lils, and a non-terminal or a terminal symbol in R.IIS.
} 
The LHS term of an arc means the LHS term of the base rule of the arc, and RHS terms of an arc means RHS terms of the base rule of the arc. In the above notation, a single dot indicates that RHS terms located to the left of a dot are inactive, that is, they already match the LHS term of some other arcs. Three dots are used to represent zero or any number of terms. An arc whose RHS terms are all inactive is called an inactive arc, otherwise it is called an active arc. An arc covers a segment of input words; the start point of an arc is the index of the first word in the covering segment, and the end point of an arc is 1 plus the index of the last word in the covering segment.

Basically, a standard CFG parsing algorithm such as $[3,6]$ consists of the following three opcrations.

Initialization: For cach word, arcs are generated from rules such that the leftmost RHS term matches it.

Operation A: For each inactive $\operatorname{arc} A$, an $\operatorname{arc}$ is generated from $A$ and a rule $R$ such that the leftmost RHS term of $R$ matches the LHS term of $\mathrm{A}$.

Operation B: For each inactive $\operatorname{arc} \mathrm{A}$, an $\operatorname{arc}$ is generated from $A$ and another active arc $B$ such that the leftmost active RHS torm of $B$ matches the LHS term of A and the end point, of $B$ is the same as the start point of $A$.

We assume that some dependency information between words are given, and such dependency information is denoted as follows:

$$
\begin{aligned}
& W_{x} \Leftarrow W_{y} \\
& W_{x} \Rightarrow W_{y}
\end{aligned}
$$

The first of the above examples represents that a word $W_{y}$ modifics another word $W_{x}$ and $W_{x}$ precedes $W_{y}$, while the second one represents that a word $W_{x}$ modifies another word $W_{y}$ and $W_{x}$ precedes $W_{y}$.

Given this kind of dependency information, the following conditions are imposed on Operation A and Operation B.

Conditions for Operation A:

Condition A1 (when the leftmost RHS term of a rulc is a head term):

Given an inactive arc $A r c_{I}$ denoted by $[A \rightarrow \ldots]$ and a rule which has two or more RHS terms and the leftmost RHS term is a head denoted by $\{X \rightarrow A *$ $B \ldots\}$, Opcration A is executed only if there is dependency information $W_{a} \Leftarrow$ $W_{b}$ where $W_{a}$ is a word matching the LHS term $A$ of $A r c_{I}$ and $W_{b}$ is a word located anywhere to the right of the end point of $A r c_{I}$.

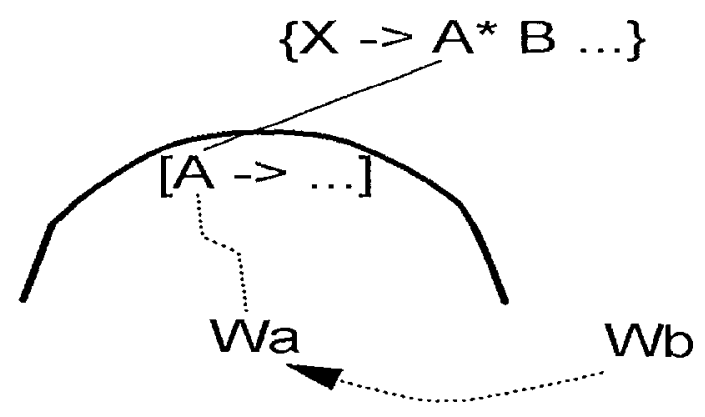

Figure 1: Condition A1

Figure 1 shows the above condition. In this figure, a thick arc represents an inactive arc, a line represents a matching to be tried in this operation, a dotted line represents a matching between a term in an arc and a word, and a dotted arrow represents dependency information. In this case, this type of rule implies that a word matching the LHS term of the arc to be matched with the leftmost term of the rule must be modified by any word which is located after the end point of the arc, since the head term is the leftmost term of the rulc. Therefore, if the A1 condition does not hold, Operation A is not required to be executed.

Condition A2 (when the leftmost RHS term of a rule is not a head torm):

Given an inactive arc $A r c_{I}$ denoted by $[A \rightarrow \ldots]$ and a rule which has two or more RHS terms and the leftmost RHS term is not a head denoted by $\{X \rightarrow A$ ... $D * \ldots\}$, Operation $\mathrm{A}$ is executed only if there is a dependency information $W_{a} \Rightarrow W_{b}$ where $W_{a}$ is a word matching the LHS term $A$ of $A r C_{I}$ and $W_{b}$ is a word located anywhere after the end point of $A r c_{I}$.

Figure 2 shows the above condition. In this case, this type of rule implies that a word matching the LHS term of the arc to be matched with the leftmost term of the rule must modify any word which is located after the end point of the arc, since the head term is not the leftmost term of the rulc.

Conditions for Operation B:

Condition $\mathrm{B} 1$ (when the leftmost active RHS term of an active arc is the head term):

Given an active $\operatorname{arc} A r c_{A}$ denoted by $\left[X \rightarrow A_{0} \ldots A_{n} . B * \ldots\right]$ and an inactive arc $A r c_{I}$ denoted by $[B \rightarrow \ldots]$ 


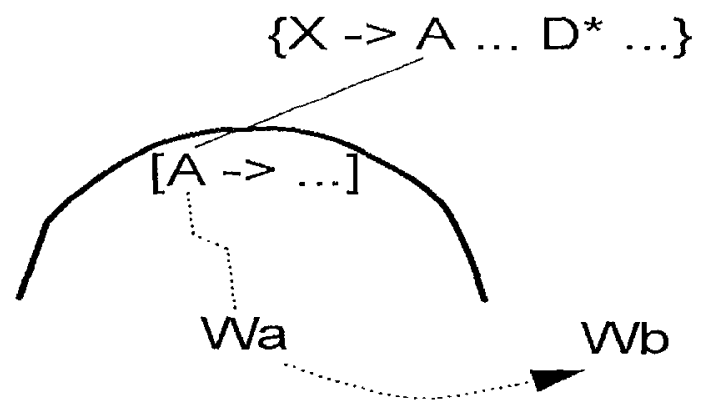

Figure 2: Condition A2

such that the end point of $A r c_{A}$ is the samo as the start point of $A r_{I}$, Operation $\mathrm{B}$ is executed only if, for each $W_{a i}$ $(0 \leq i \leq n)$ which is a word matching the RHS torm $A_{i}$ of $A r c_{A}$, there is dependency information $W_{a i} \Rightarrow W_{b}$, where $W_{b}$ is a word matching the LHS term $B$ of $A r c_{I}$.

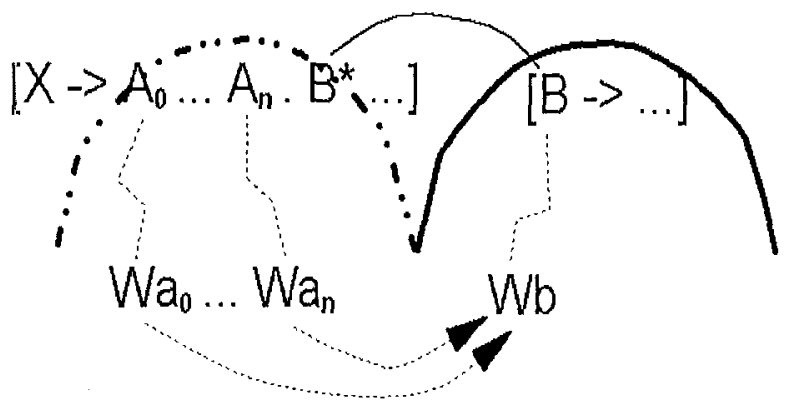

Figure 3: Condition B1

Figure 3 shows the above condition. In this figure, a dotted thick are represents an active arc. In this case, this type of active are implics that words matching inactive terms before the head term of the active are must modify a word matching the LHS term of the inactive arc.

Condition B2 (when the head term is on the left side of the leftmost active RHS torm of an active arc):

Given an active $\operatorname{arc} A r c_{A}$ denoted by $[X \rightarrow \ldots A * \ldots . . B \ldots]$ and an inactive $\operatorname{arc} A r c_{I}$ denoted by $[B \rightarrow \ldots]$ such that the end point of $A r c_{A}$ is the same as the start point of $\operatorname{Arcr}_{1}$, Operation $B$ is exccuted only if there is dependency information $W_{n} \Leftarrow W_{b}$ where $W_{a}$ is a word matching the RHS term
$A$ of $A r C_{A}$, and $W_{b}$ is a word matching the LuHS term $B$ of $A r_{I}$.

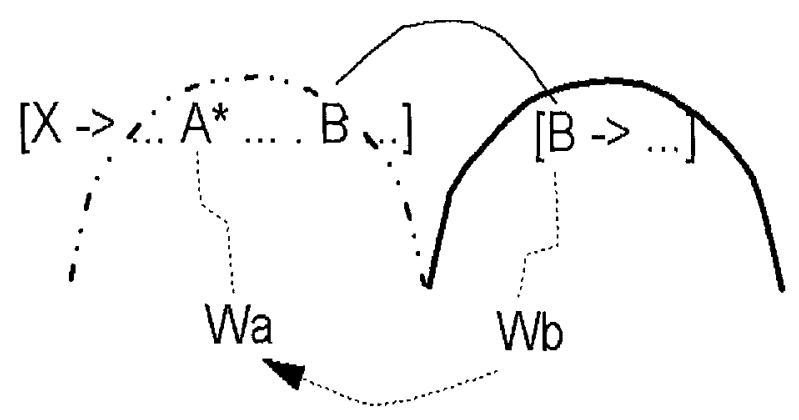

Figure 4: Condition B2

Figure 4 shows the above condition. In this case, this type of active arc implies that a word matching the LHS term of the inactive arc must modify a word matching the head term of the active arc.

Condition B3 (when the head term is on the right side of the leftmost active RHS term of an active arc):

Given an active $\operatorname{arc} A r c_{A}$ denoted by $[X \rightarrow A . B \ldots C * \ldots]$ and an inactive arc $A r c_{I}$ denoted by $[B \rightarrow \ldots]$ such that the end point of $A r C_{A}$ is the same as the start point of $\mathrm{Arc}_{1}$, Operation B is exccuted only if there is dependency information $W_{b} \Rightarrow W_{c}$ whore $W_{b}$ is a word matching the LHS term $B$ of $A r^{2} C_{I}$, and $W_{c}$ is a word on the right side of the end point of $A r c_{I}$.

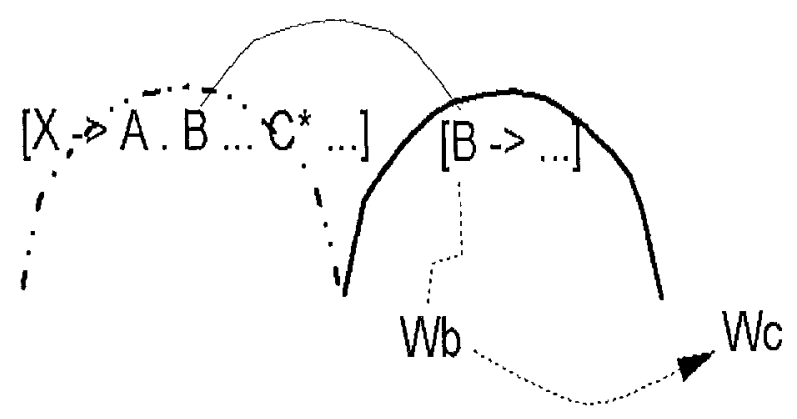

Figure 5: Condition $B 3$

Figure 5 shows the above condition. In this case, this type of active arc implies that a word matching the LHS term of the inactive are must modify a word after the end point of the inactive arc.

The dependency information is not necessarily given to all words. If there is any source word except for the root word of a sentence such that there 
is no dependency information originating from it, then a set of such depondency information is called partial, otherwise, it is called total. If the given dependency information is partial, the Al condition can not be used, since, even if there is no dependency information targeting $W_{a}$, we cannot know if such dependency information does not really exist, or if such dependency information is not suppliod. For other conditions, we check them only when all source words for dependency checking have dependency information. On the other hand, if the given dependency information is total, all conditions are checkod.

\section{Experiment}

We have implemented the proposed algorithm into an existing English CFG-parser we have developed for a machine translation product $[8,9,11]^{2}$ , and conducted an experiment to know the effectiveness of this algorithm.

We selected 280 test sentences landomly from a sontence sot croated by JEIDA ${ }^{3}$ for evaluating translation system, and made the correct depen. dency relation data for these selected test sentences. We collected the number of inactive ares, the number of active ares, and the processing time for cases such that $\mathrm{C}$ modifice candidates (one of which is the correct modifiec) are given to a word. ${ }^{4}$ If $\mathrm{C}=1$ then it corresponds to the best case for a parser such that only one correct modifiee is given for cach word, while if $\mathrm{C}$ is 3 or 4 then it corresponds to tho approximation of using a statistical modifice estimation program for getting candidate modifiees.

The graphs in Figure 6 indicate the reduction ratios of active arcs, inactive arcs, and processing time for using conditions for total dependency information and conditions for partial dependency information. The denominators for calculating these ratios are the numbers of ares and the processing time (seconds) in case of the parser: without this algorithm. In these graphs, $\mathrm{C}=\mathrm{X}$ indicates that $\mathrm{X}$ is the maximum number of modifice candidates given to a word.

From these graphs, we can see that the moro words in a sentence, the better the performance. In a real domain, most sentences consist of more than ten words. Therefore, looking at values for around 10 in the $\mathrm{X}$ axis, we can sce that inactive ares are reduced by about $40 \%$ and $25 \%$, active arcs

\footnotetext{
${ }^{2}$ This parser is used in a Web page translation software called "Internet King of Thanslation" released from IIBM Japan.

${ }^{3}$ Japan Electronic Industry 1)evelopment Association

${ }^{4}$ Modifiec candidates are selected randomly except for the correct one.
}

are reduced by about $65 \%$ and $35 \%$, and processing time is reduced by about $45 \%$ and $15 \%$, for the idcal case $(\mathrm{C}=1)$ and more practical cases $(C=3$ or 4), respectively, in the case of total dependency information. Please note that, since the parser in which this algorithm is implomented has already several pruning mechanisms, we can expect more reduction (or performance gain) for gencric CFG parsers.

\section{Discussion}

As a study for accelerating the parsing process using dependency information, Imaichi[5] reported an algorithm for Japanese language. The conditions introduced by Imaichi are described by using the notation in this paper as follows:

Condition M1:

Given an active arc $A r c_{A}$ denoted by $[X \rightarrow A . B *]$ and an inactive are $A r^{*} c_{1}$ denoted by $[B \rightarrow \ldots]$ such that the end point of $A r c_{A}$ is the same as the start point of $A r c_{I}$, Operation $B$ is executed only if there is dependency information $W_{a} \Rightarrow W_{b}$ where $W_{a}$ is a word matching the RIIS term $A$ of $A r c_{A}$, and $W_{b}$ is a word matching the LHS term $B$ of $A r c_{I}$.

Condition M2:

Given an inactive arc Arct donoted by $[A \rightarrow \ldots]$ and a rule denoted by $\{X \rightarrow$ $A \ldots\}$, Operation A is executed only if there is no dependency information $W_{k} \Rightarrow W_{a}$ whese $W_{a}$ is a word matching the LHS term $A$ of $A r c_{1}$ and $W_{k}$ is a word located before the start point of $\operatorname{Arc}_{1}$.

The condition M1 corresponds to 131. Since Inaichi's algorithm considers only Japanese in which all words other than the last word modifies one of the succeeding words, it does not deal with cases usually scen in European languages where a word modifies one of the preceding words. Therefore, it is not applicable to any language other than Japanese in general. Further, since a CFG rule is restricted to be in Chomsky normal form, Imaichi's algorithm is limited in terms of applicability.

Since the algorithm proposed in this paper does not have any restrictions on the dependency direction and the CFG rule format, it can be applicable to any $\mathrm{CFG}$-parsers in any languages. 


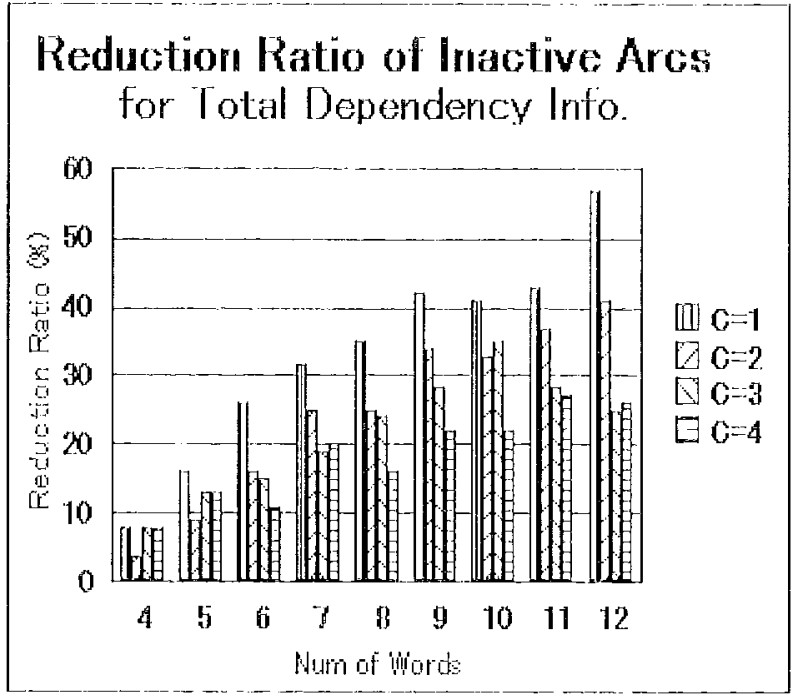

(a)

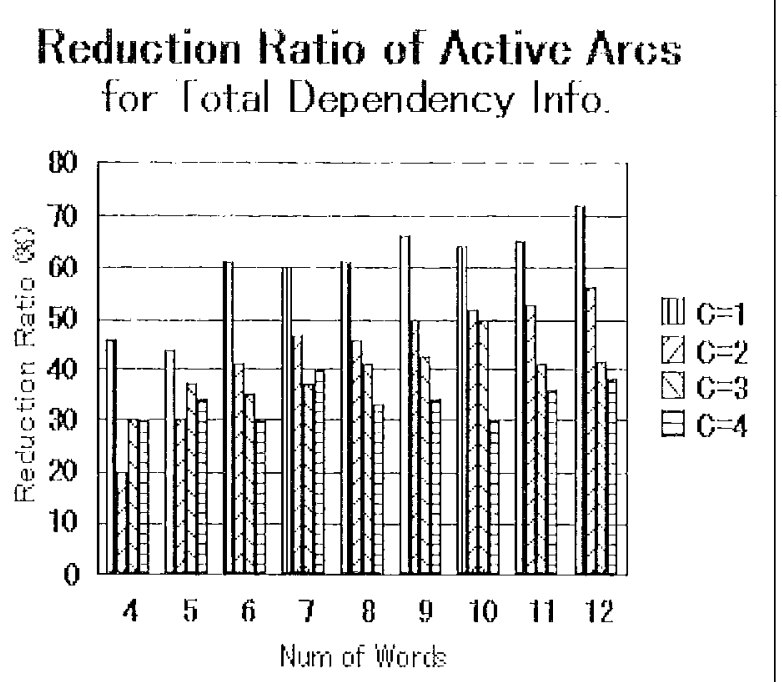

$(c)$

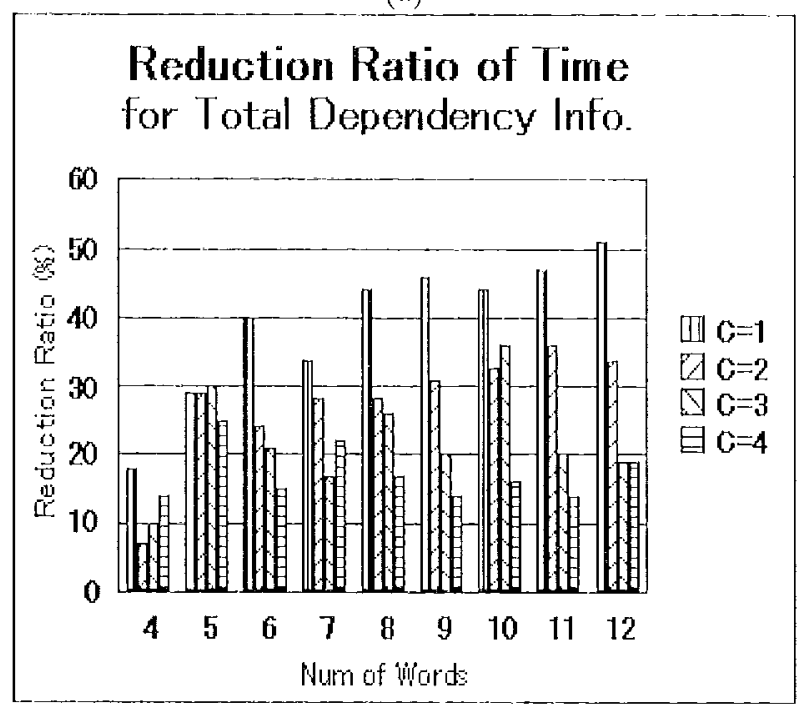

(c)

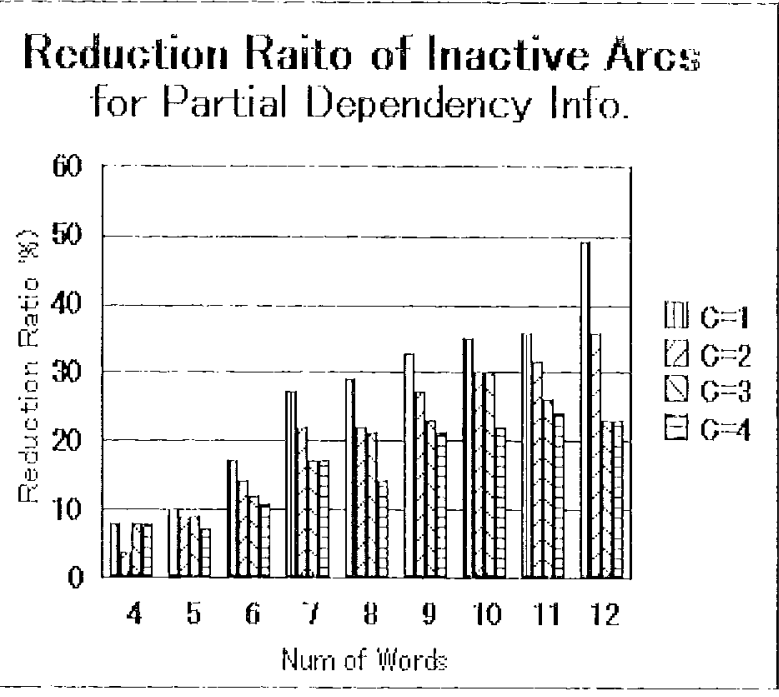

(b)

\section{Reduction Ratio of Active Arcs}

for Partial Dependency Info

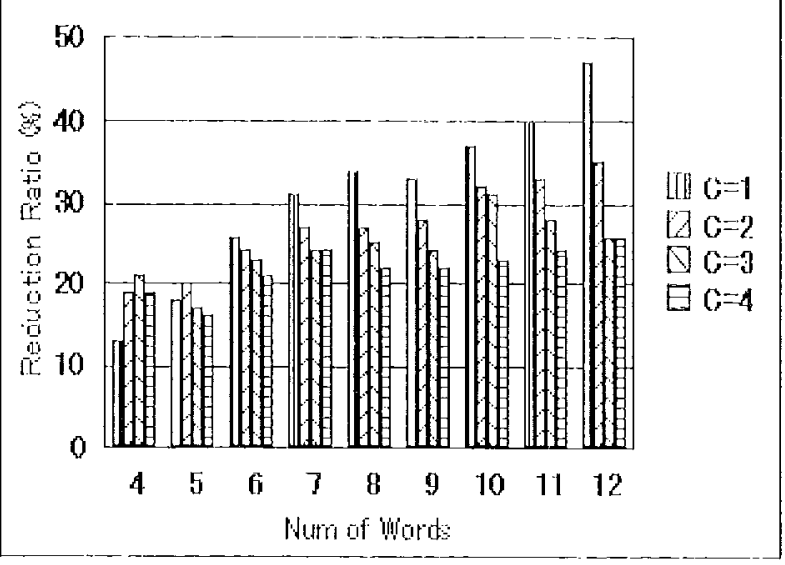

(d)

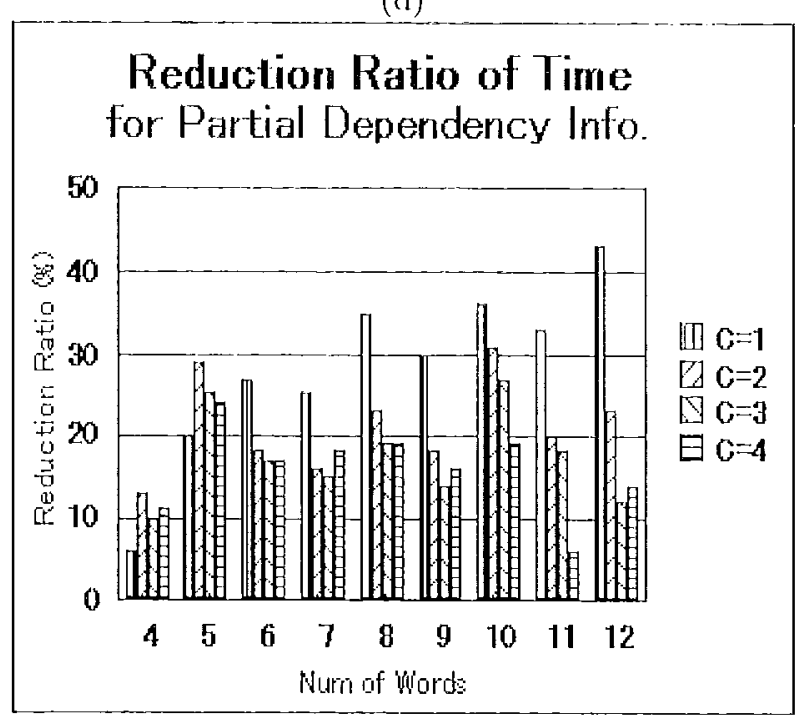

(f)

Figure 6: Reduction ratios of inactive ares, active ares, and processing time 


\section{Conclusion}

We developed an algorithm for accelerating the performance of the CFG parsing process if we are given dependency information. From an experiment, we can show the effectiveness of this algorithm.

By using this algorithm, we can enhance existing grammar-based parsers using dependency information given by stochastic parsers, interactive systems, and texts created by linguistic annotation systems.

\section{References}

[1] M. Collins. A new statistical parser based on bigram lexical dependencios. In Proc. of 34th ACL, pages 184-191, 1996.

[2] M. Collins. Three generative, lexicalized models for statistical parsing. In Proc. of 35th ACL, pages 16-23, 1997.

[3] J. Earley. An efficient context-free parsing algorithm. In Readings in Natural Language Processing. Morgan Kaufman, 1969.

[4] K. Hashida, K. Nagao, et al., Progress and Prospect of Global Document Annotation. (in Japanese) In Proc. of 4th Annual Meeting of the Association of Natural Language Processing, pp. 618-621, 1998.

[5] O. Imaichi, Y. Matsumoto, and M. Fujio. An integrated parsing method using stochastic information and grammatical constraints. Journal of Natural Language Processing, 5(3):67-83, 1998.

[6] M. Kay. Algorithm schemata and data structure in syntactic processing. Technical Report CSL-80-12, Xerox PARC, 1980.

[7] D. M. Magerman. Statistical decision-trec modcls for parsing. In Proc. of 33rd $A C L$, pages 276-283, 1995.

[8] K. Takeda. Pattern-based context-free grammars for machine translation. In Proc. of 34 th ACL, pages 144-151, 1996.

[9] K. Takeda. Pattern-based machine translation. In Proc. of 16th Coling, volume 2, pages 1155$1158,1996$.

[10] Text Encoding Initiative (http://www.uic.edu:80/orgs/tei/)

[11] H. Watanabe and K. Takeda. A patternbased machine translation system extended by example-based processing. In Proc. of 17 th $\mathrm{Col}$ ing (Coling-ACL'98), volume 2, pages 13691373, 1998.
[12] H. Watanabe, Linguistic Annotation Language - The Markup Language for Assisting NLP Programs -. IBM Research Report RT0334, 1999.

[13] H. Watanabe, K. Nagao, et al., Linguistic Annotation System for Improving the Performance of Natural Language Processing Programs. In Proc. of 6th Annual Meeting of The Association for NLP (in Japanesc), pp. 171-174, 2000. 